\title{
Glass Syringe
}

National Cancer Institute

\section{Source}

National Cancer Institute. Glass Syringe. NCI Thesaurus. Code C43203.

A device for the administration of parenteral drug products that consists of a rigid glass

barrel fitted with septum with a plunger at one end and a seal or needle at the other end.

The needle assembly may be part of the device or separate. 